\title{
riccafd
}

Revista Iberoamericana de Ciencias de la Actividad Física y el Deporte

\section{NEUROCIENCIA Y ENTRENAMIENTO EN EL DEPORTE DE ALTO RENDIMIENTO}

\section{NEUROSCIENCE AND TRAINING IN HIGH PERFORMANCE SPORTS}

\author{
Alfonso Mantilla José Iván \\ Fisioterapeuta, Universidad del Rosario, Colombia, josealfonso25@hotmail.com
}

Correspondencia: Alfonso Mantilla José Iván. josealfonso25@hotmail.com

Código UNESCO: 321311. Fisioterapia

Clasificación Consejo de Europa: 14. Fisioterapia y Rehabilitación

Recibido el 10 de octubre de 2018

Aceptado el 22 de abril de 2019

DOI: http://dx.doi.org/10.24310/riccafd.2019.v8i2.6238

\section{RESUMEN}

El objetivo de este estudio fue realizar una revisión sistemática de la literatura en relación a la neurociencia aplicada al deporte de alto rendimiento. Esta se realizó a través de una búsqueda en bases de datos con palabras clave como percepción visual, tiempo de reacción, tiempo de ejecución y deporte. Se identificó que existen factores mentales que determinan el éxito del deportista de alto rendimiento, estos están mediados por aspectos psiconeurofisiologicos del sistema nervioso que incrementan la actividad neuronal facilitando el aprendizaje y adaptación de gestos motores a circunstancias específicas del deporte. Como resultados, en primera instancia se determinó que existen habilidades cognitivas que son determinantes en la consecución de logros deportivos. Estas son atención sostenida, memoria verbal, velocidad de procesamiento y memoria visoespacial. Adicionalmente, existen metodologías de entrenamiento para estas habilidades que se desarrollan a través de dispositivos tecnológicos, juegos de realidad virtual y entrenamiento en campo. En conclusión, el entrenamiento de habilidades cognitivas es el camino para aumentar el rendimiento mental del deportista y generar una mejor toma de decisiones en el campo de juego que puede ser vital en la consecución de logros deportivos por parte de entidades deportivas a nivel nacional e internacional.

Palabras clave: percepción visual, tiempo de reacción, tiempo de ejecución, deporte. 


\section{ABSTRACT}

The objective of this study was to conduct a review of the literature in relation to neuroscience applied to high performance sports. This was done through a database search with keywords such as visual perception, reaction time, execution time and sport. It was possible to see that there are mental factors that determine the success of the high performance athlete. Psychoneurophysiological aspects of the nervous system that increase neuronal activity, facilitating the learning and adaptation of motor gestures to specific sport circumstances, mediate these. As results, in the first instance it was determined that there are cognitive abilities that are determining in the achievement of sporting achievements. These are sustained attention, verbal memory, processing speed and visuospatial memory. In the second instance, it was determined that there are training methodologies for these skills that can be through technological devices, virtual reality games and field training. In conclusion, the training of cognitive skills is the way to increase the mental performance of the athlete and generate better decision making on the field of play that can be vital in the achievement of sporting achievements by sports entities at national and international level.

Key words: Visual perception; reaction time; execution time; sport.

\section{INTRODUCCIÓN}

En la actualidad, el deporte de alto rendimiento se ha convertido en un foco de estudio por parte de centros especializados en medicina deportiva alrededor del mundo. Distintos profesionales en áreas de la salud y entrenamiento deportivo trabajan conjuntamente para obtener distinciones deportivas a nivel nacional e internacional con diferentes entidades del deporte. El éxito deportivo esta mediado por factores multifactoriales como condiciones físicas, técnicas, tácticas y psicológicas que llevaran al deportista de alto rendimiento a ser considerado una unidad funcional a nivel fisiológico, físico y cognitivo(1,2). Los profesionales que trabajan en alto rendimiento, utilizan diferentes metodologías de entrenamiento para lograr potencializar las capacidades del deportista. Para ejemplificar, preparación física, fisioterapia y psicología trabajan conjuntamente en la prevención de lesiones, recuperación e incremento del estado físico y mental de deportistas(3-5).

La psicología deportiva estudia componentes mentales internos y externos implicados en el rendimiento deportivo. El área de psicología trabaja en conjunto con los demás profesionales en la preparación del deportista a través de la neurociencia deportiva, la cual comprende aspectos psiconeurofisiologicos del aprendizaje motor mediados por cambios biológicos, químicos y morfológicos que incrementan la actividad neuronal facilitando la apropiación de gestos motores a circunstancias específicas del deporte. Por ejemplo, las neuronas espejo juegan un papel fundamental en la retroalimentación de acciones de juego mediante la activación de áreas cerebrales como los lóbulos frontales y cerebelo que garantizan la mecanización de un gesto motor especifico respondiendo a estímulos directos del juego(5-8). 
El trabajo en conjunto entre todos los profesionales en equipos deportivos garantiza el aumento del rendimiento deportivo a nivel global del deportista. De hecho, los profesionales cada día buscan diferentes maneras de potencializar las habilidades físicas y cognitivas de deportistas de alto rendimiento. Por ejemplo, psicólogos realizan entrenamientos para aumentar procesos cognitivos como atención, reacción, memoria, confianza y autocontrol para incrementar el estado neurocognitivo de deportistas en diferentes disciplinas deportivas(5, 9).Por tal motivo, el objetivo de este artículo es realizar una revisión de la literatura en relación a la importancia del trabajo neurocognitivo en deportistas de alto rendimiento y una propuesta de trabajo en campo.

\section{MATERIAL Y METODOS}

Se realizó una revisión sistemática de literatura donde se determinaron los siguientes criterios de inclusión y exclusión:

Inclusión: En primera instancia, se estableció como primer criterio estudios contemplados del año 2000 al 2018, literatura que contemplara los siguientes términos $\mathrm{MeSH}$ : Visual perception, reaction time, execution time, neuroscience and sport. La literatura se aceptaría en idioma español, alemán, inglés y portugués

Se estableció como otro criterio de inclusión que la búsqueda de evidencia se basará en: Fuentes primarias: Estudios ECA, Meta Análisis, casos y controles, estudios de cohorte, revisiones sistemáticas, revisiones de literatura. Fuentes secundarias: Monografías, tesis de grado, libros.

Exclusión: Estudios que su año de publicación fuera inferior al año 2000, que no contemplaran los términos MeSH establecidos, estudios que no estuvieran disponibles o completos

\section{RESULTADOS}

En la figura 1 se muestra el proceso de extracción de la evidencia identificada en las bases de datos y su proceso de selección

\section{Habilidades Neurocognitivas}

Dentro de la revisión, se identificó que las habilidades cognitivas que deben ser trabajadas por los deportistas en campo son: atención sostenida definida como actividades atencionales consientes sobre una actividad específica como por ejemplo estado de alerta y observación(10,11). Memoria verbal, la cual es la capacidad de almacenar información de tipo verbal, táctica y conceptual(12). Memoria visoespacial definida como la capacidad de memorizar objetos, personas en un determinado lugar y situación $(13,14)$. Velocidad de procesamiento definida como la capacidad de relacionar la información y sacar una conclusión de manera efectiva(15, 16). Es así como se determinó en la figura 2 las habilidades cognitivas implicadas en el campo de juego y en la figura 3 la relación del proceso de retroalimentación sensorial. 


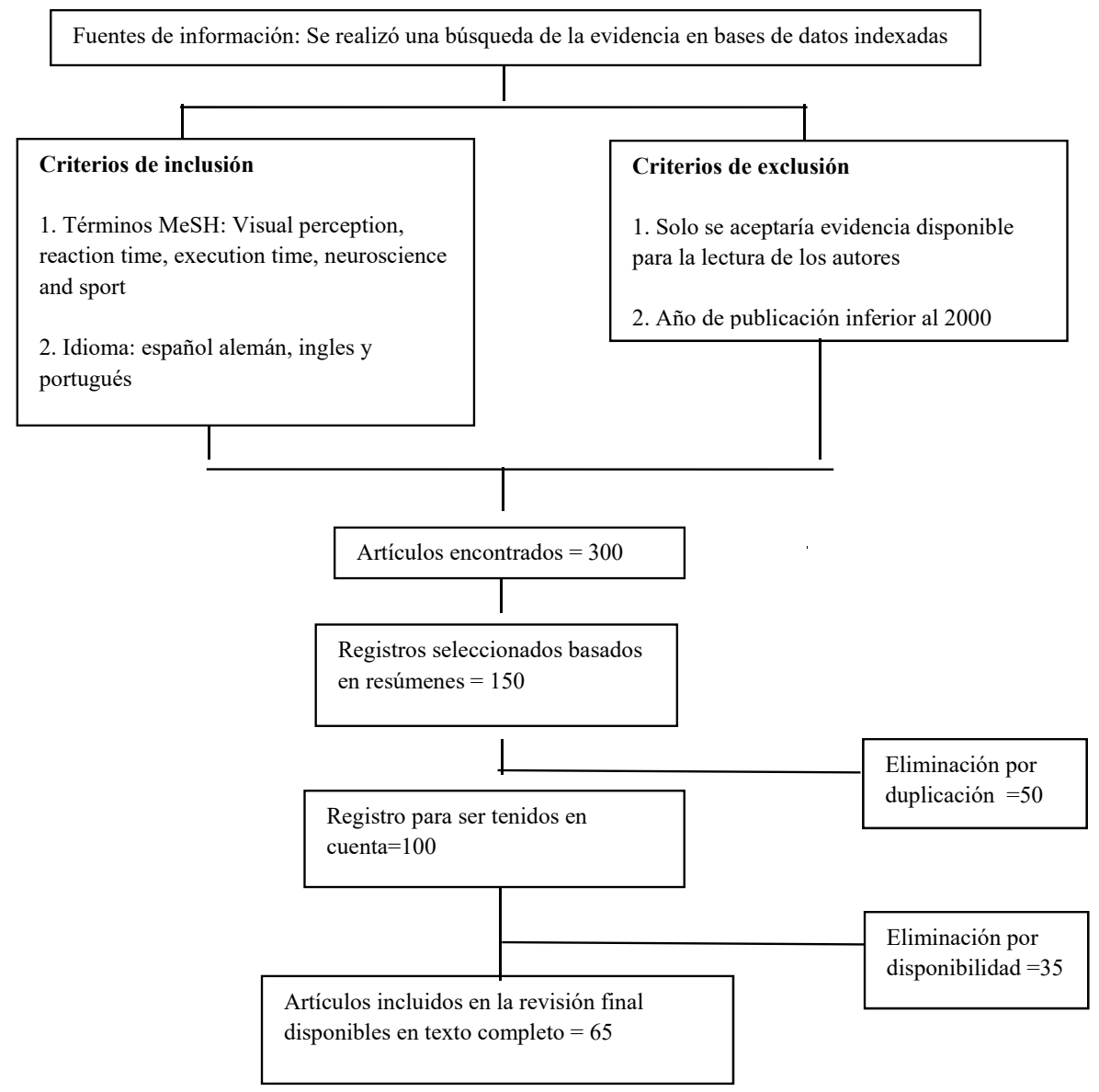

Figura 1. (Flujograma de extracción de la evidencia).

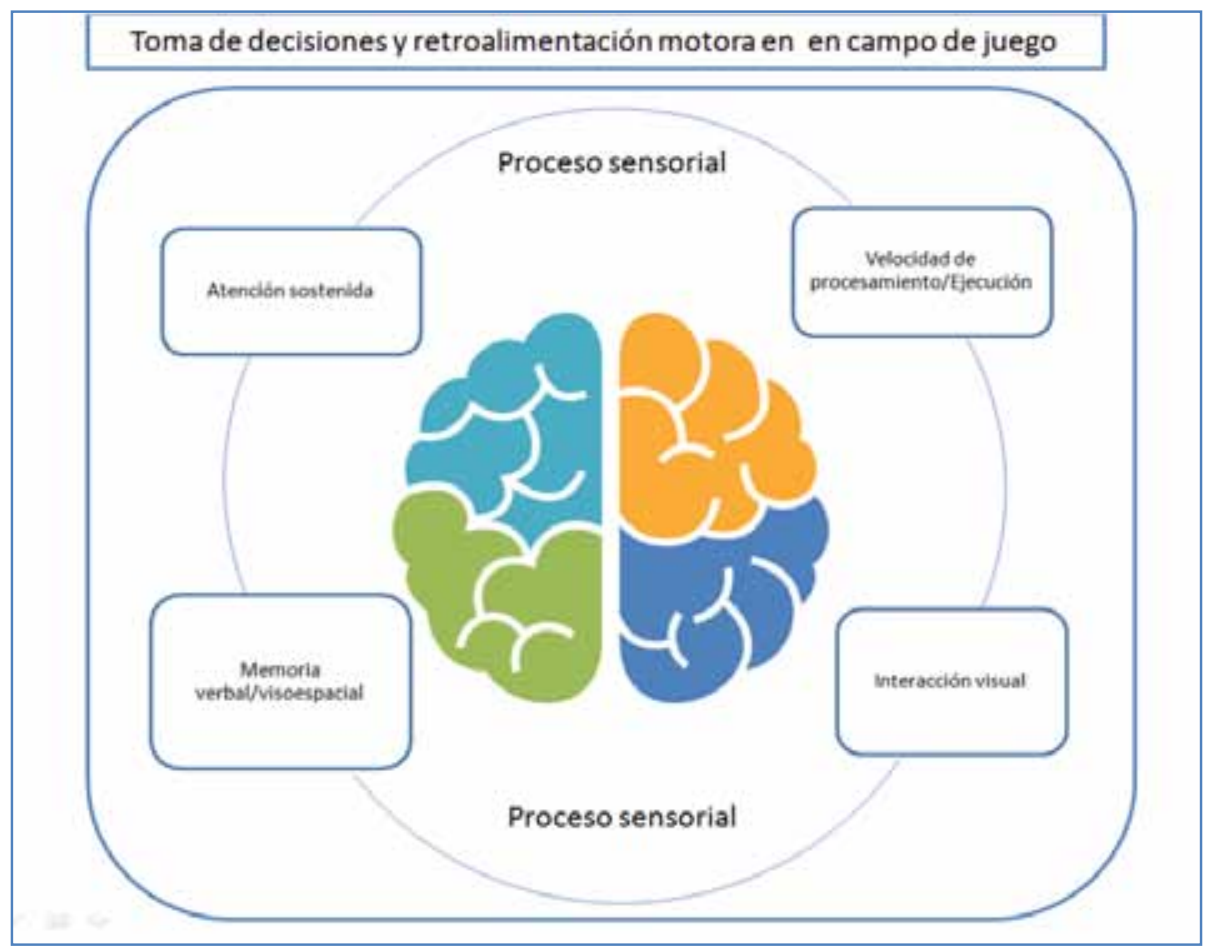

Figura 2. (Habilidades cognitivas en campo de juego); Fuente de elaboración propia 2017.

\begin{tabular}{l|l}
\hline 82 & NEUROCIENCIA Y ENTRENAMIENTO EN EL DEPORTE \\
DE ALTO RENDIMIENTO
\end{tabular}




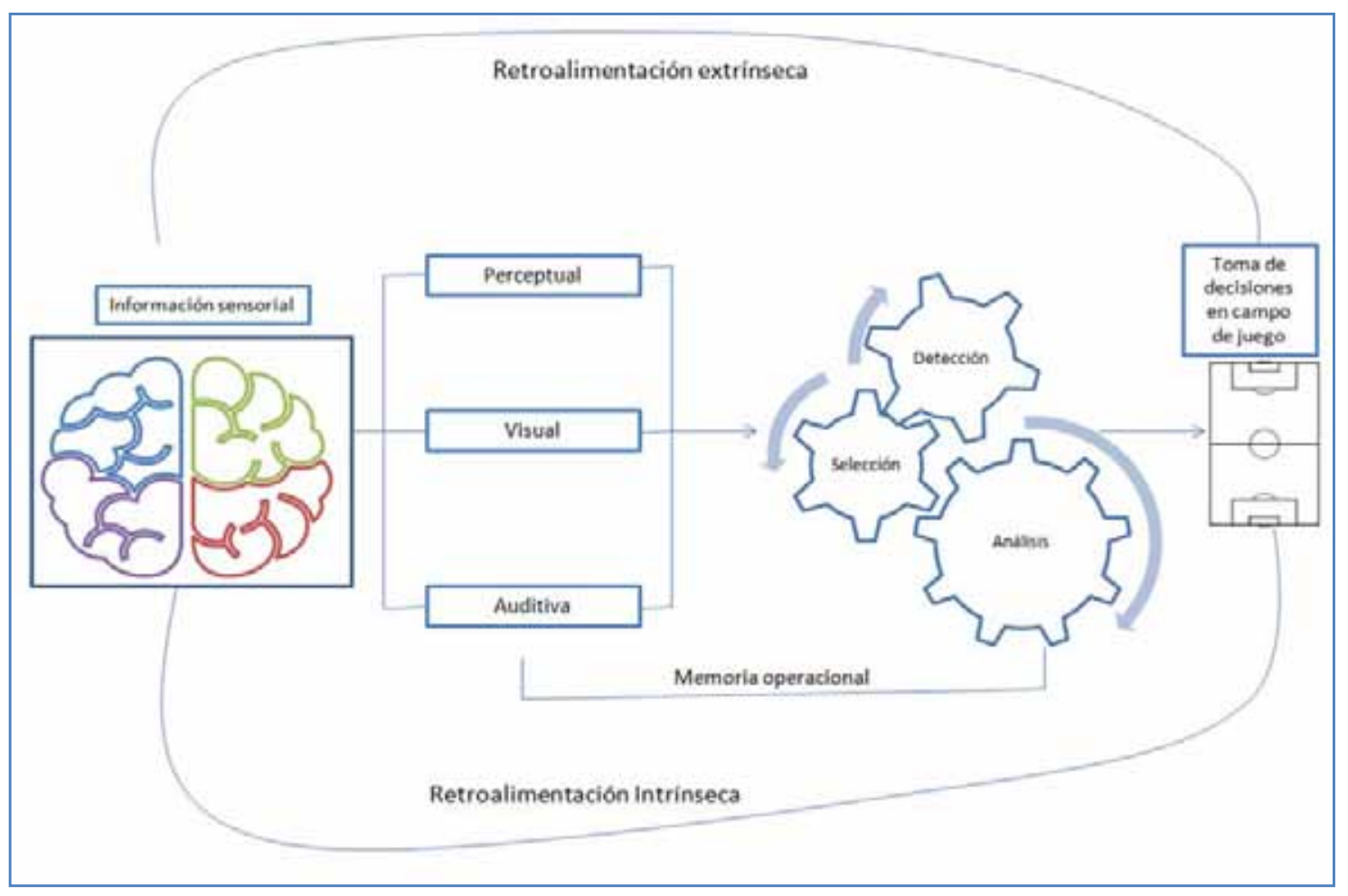

Figura 3. (Proceso de retroalimentación sensorial para la toma de decisiones en campo de juego); Fuente de elaboración propia 2017

\section{Dispositivos tecnológicos de entrenamiento cognitivo}

Existen dispositivos de entrenamiento cognitivo enfocados en el aumento de habilidades sensoriales en deportistas como la agudeza visual, visión periférica, velocidad de reacción y coordinación oculomotora los cuales aumentan la velocidad de procesamiento de información en relación a la toma de decisiones en campo de juego. Entre los más conocidos a nivel mundial se encuentran: Dispositivo Neurotracker 3D-MOT el cual permite el entrenamiento de la agudeza, coordinación visomotora y velocidad visual(9, 17-22). Dispositivo footbonaut y Helix los cuales son dispositivos específicos para el trabajo de visión periférica, precisión y agilidad mental en futbolistas (23-26). Dispositivos de entrenamiento visual Impulse Strobe Glasses, Nike SPARQ Vapor Strobe Eyewear, I-ON Training Glasses, Eyeport, Dynavision D2 los cuales aumentan la capacidad cognitiva para la captación de estímulos visuales mejorando la velocidad de procesamiento entre la información visual y su reacción en campo de juego(27-37). Dispositivos Octopus Trainer y fitLight Trainer ${ }^{\mathrm{TM}}$ los cuales enfocan su trabajo en el entrenamiento de la velocidad de reacción y percepción visual aumentando la retroalimentación en campo de juego utilizando diferentes tipos de estímulos visuales(9, 38-40). Dispositivo Batak enfocado en el entrenamiento de velocidad de reacción y visión(41, 42). Dispositivos Sports Vision Trainer (SVTTM), Wayne Saccadic Fixator , Wayne Peripheral Awareness PAT, AcuVision 2000 Makoto Arena II y Sicropat los cuales aumentan la coordinación visomotora y retención visual(43-52) 


\section{Juegos y simuladores de realidad virtual en deporte}

Se utilizan juegos y simuladores de realidad virtual para trabajar habilidades como atención y memoria que determinan la relación que existe entre la velocidad de procesamiento y la toma de decisiones en el terreno de juego. Entre los más conocidos se encuentran: Vizual Edge Performance Trainer (VEPT), HawkEye Sports Simulators, SPEESION, Sanet Vision Integrator (SVI) y Nike SPARQ Sensory Training Station(9, 53-60).

\section{Entrenamiento en campo}

Se pudo determinar que existen factores específicos ante el entrenamiento neurocognitivo determinado por condiciones de los deportistas. Por ejemplo, la velocidad de reacción no está relacionada con los años de práctica del deporte debido a que es una capacidad del sistema nervioso determinado por factores genómicos. Por el contrario, la velocidad de ejecución se relaciona con el tiempo de entrenamiento del deportista. Otro factor de análisis es la fatiga fisiológica debido a que está se asocia a disminución de calidad en la ejecución de gestos motores y déficit atencionales en campo de juego(61). El entrenamiento neurocognitivo debe estar siempre presente en las sesiones de preparación física con el fin de potencializar no solamente la parte física sino también la mental. Para dicho objetivo se deben utilizar metodologías de entrenamiento basados en estímulos visuales, memoria, percepción sensorial trasladado al campo de juego con ejercicios con diferentes elementos. Para ejemplificar, arqueros realizan trabajos de diferenciación con respecto a la trayectoria y velocidad del balón ante diferentes situaciones para entrenar la agudeza visual y el tiempo de reacción(61-64). Adicionalmente, se realizan trabajos específicos con diferentes estímulos como colores y órdenes verbales creando relaciones cíclicas de información generando una asociación entre el sistema sensorial y el sistema motor permitiendo desarrollar inteligencia táctica en jugadores profesionales(65).

\section{DISCUSIÓN}

En el deporte de alto rendimiento, el cuerpo técnico y medico deben trabajar de manera conjunta y planificada para lograr potencializar las capacidades de los jugadores en aspectos físicos, mentales y tácticos. Esto se logra a través de trabajos específicos diseñados dentro del plan de trabajo establecido por parte del cuerpo técnico. De hecho, el trabajo neurocognitivo debe ser un pilar dentro de la planificación semestral y anual en todo equipo deportivo debido a que permite definir con exactitud la diferencia entre la capacidad de toma de decisiones entre los jugadores que es vital en la consecución de títulos a nivel nacional e internacional. Es necesario que se adapten trabajos neurocognitivos para ser desarrollados en campo o en laboratorio debido a que estos mejoran los procesos de detección de talento deportivo e incrementan habilidades cognitivas en jugadores con el fin de mejorar el estilo de juego y así poder generar venta 
de jugadores a nivel internacional y nacional. Entidades nacionales dedicadas a investigación deben desarrollar tecnologías de bajo costo y accesibles a equipos deportivos que permitan el entrenamiento cognitivo de deportistas mejorando procesos de entrenamiento individual y grupal que generan adaptaciones a nivel mental en el deportista.

\section{Propuesta de protocolo}

Con la información obtenida, en la tabla 1 se realiza una propuesta de protocolo de trabajo en campo para deportistas como futbolistas de alto rendimiento.

\section{Trabajo en campo}

- Trabajos de memoria visual con colores, números y objetos.

- Trabajos de velocidad de reacción con balones de diferentes tamaños, pesos y colores

- Trabajos de percepción de la trayectoria del balón con elementos como discos, platillos y pelotas de diferentes tamaños lanzados a una altura determinada

- Ejercicios de atajadas con diferentes tipos de balón y expuesto a diferentes situaciones de juego como rebotes los cuales pueden ser simulados con trampolines, cajas, tapetes con agua.

- Ejercicios en corta distancia con diferenciación realizada con bombas, pelotas de diferentes tamaños finalizando con gesto motores específicos.

- Ejercicios sobre cambio de dirección del balón los cuales pueden ser desarrollados con conos y estacas.

- Ejercicios de distorsión cognitiva con dos balones para mejorar el dominio y control de balón

- Trabajos de orden táctico en retrocesos defensivos con ayuda de conos, colores y memoria verbal.

- Trabajos de despeje de balón con pelotas de distintos tamaños

- Trabajos de orientación y salida con ayuda de colores y memoria visual

- Ejercicios de salida bajo presión con ayuda de colores.

- Trabajos de salidas desde el medio campo con apertura hacia bandas y extremos con ayuda de colores y órdenes verbales.

- Trabajos de marcaje con ayuda de conos, colores y aros

- Ejercicios de definición al arco con diferentes tamaños de pelotas y arcos de diferentes tamaños

- Ejercicios de percepción visual y memoria visoespacial enfocando el trabajo en pase, recepción y definición

- Ejercicios en bases inestables con relación cognitiva con bombas, números y balones 


\section{Conclusión}

El entrenamiento neurocognitivo permite incrementar habilidades visuales, perceptivas y visoespaciales desarrollando adaptaciones neuronales en deportistas mejorando la toma de decisiones en el campo de juego incrementando las posibilidades de la obtención de logros deportivos a nivel nacional e internacional. se deben desarrollar programas específicos de entrenamiento en equipos deportivos para realizar mediciones de la efectividad de programas de entrenamiento neurocognitivo.

\section{REFERENCIAS}

1. Williams CA, James DV. Science for exercise and sport: Psychology Press; 2001.

2. Cabezas MM, Córdoba EP, Manzano JMG, Benítez MAG, Loquiño JES, Martín MV. Planificación psicológica de la cantera del Sevilla FCSAD: organización, funcionamiento y programa deportivo-formativo. Cuadernos de Psicología del Deporte. 2003;3(2).

3. Cometti G. La preparación física en el fútbol: Editorial Paidotribo; 2007.

4. Barcelona FC, Mèdics S. Guía de Práctica Clínica de las lesiones musculares. Epidemiología, diagnóstico, tratamiento y prevención. Versión 4.5 (9 de febrero de 2009). Apunts: Medicina de l'esport. 2009;44(164):179-203.

5. García-Naveira A. El psicólogo del deporte en el alto rendimiento: aportaciones y retos futuros. Papeles del Psicólogo. 2010;31(3).

6. Tamorri S. Neurociencias y deporte. Psicología deportiva. Procesos mentales del atleta: Editorial Paidotribo; 2004.

7. Reyes A. La implicación de la neurociencia en la ejecución gimnástica. Sapiens Revista Universitaria de Investigación. 2009;10(1):179-201.

8. Cox RH. Psicología del deporte: conceptos y sus aplicaciones: Ed. Médica Panamericana; 2008.

9. Appelbaum LG, Erickson G. Sports vision training: A review of the state-of-the-art in digital training techniques. International Review of Sport and Exercise Psychology. 2016:1-30.

10. Sastre-Riba S, Viana-Sáenz L. Funciones ejecutivas y alta capacidad intelectual. 2016.

11. Bartés i Serrallonga M, Adan A, Solé-Casals J, Caldú i Ferrus X, Falcón C, Pérez Pàmies $\mathrm{M}$, et al. Bases cerebrales de la atención sostenida y la memoria de trabajo: un estudio de resonancia magnética funcional basado en el Continuous Performance Test. 2014.

12. Takashima A, Bakker I, Van Hell JG, Janzen G, McQueen JM. Interaction between episodic and semantic memory networks in the acquisition and consolidation of novel spoken words. Brain and language. 2017;167:44-60.

13. Ramirez W. Niveles de funcionamiento neuropsicológicos: atención, memoria y capacidad intelectual en jugadores de baloncesto. Lecturas Educacion Fisica y Deportes, Revista Digital: efdeportes com. 2003;9(66). 
14. Guzmán-Cortés J, Villalva-Sánchez A, Bernal J. Cambios en la estructura y función cerebral asociados al entrenamiento aeróbico a lo largo de la vida. Una revisión teórica. Anuario de Psicología. 2015;45(2).

15. Alvarez JA, Emory E. Executive function and the frontal lobes: a meta-analytic review. Neuropsychology review. 2006;16(1):17-42.

16. Carlson SM. Executive function in context: Development, measurement, theory, and experience. Monographs of the Society for Research in Child Development. 2003;68(3):138-51.

17. Junyent LQ, Blázquez AP, Fortó JSI, Torradeflot GC. Entrenamiento perceptivocognitivo con el Neurotracker 3D-MOT para potenciar el rendimiento en tres modalidades deportivas/Perceptual-cognitive Training with the Neurotracker 3D-MOT to Improve Performance in Three Different Sports. Apunts Educació física i esports. 2015(119):97.

18. Beauchamp P, Faubert J. Visual Perception Training: Cutting Edge Psychophysics and 3D Technology Applied to Sport Science. High Performance CIRCuit-e-Journal. 2011;1(3):12-6.

19. Grushko A, Bochaver K, Shishkina A, Kabanov D, Konstantinova M, Vavaev A, et al. Psychological and psychophysiological profile in combat sports. Revista de Artes Marciales Asiáticas. 2016;11(2s):70-1.

20. Faubert J. Professional athletes have extraordinary skills for rapidly learning complex and neutral dynamic visual scenes. Scientific reports. 2013;3:1154.

21. Romeas T, Guldner A, Faubert J. 3D-Multiple Object Tracking training task improves passing decision-making accuracy in soccer players. Psychology of Sport and Exercise. 2016;22:1-9.

22. Mangine GT, Hoffman JR, Wells AJ, Gonzalez AM, Rogowski JP, Townsend JR, et al. Visual tracking speed is related to basketball-specific measures of performance in NBA players. The Journal of Strength \& Conditioning Research. 2014;28(9):2406-14.

23. Saal C, Müller S, Fiedler H, Mayer J, Lanwehr R. The Footbonaut as an Innovative Diagnostic System. 2014.

24. Dupré T, Mortensen KR, Lysdal FG, Funken J, Müller R, Braun M, et al., editors. Hip Joint Load and Muscle Stress in Soccer Inside Passing. ISBS-Conference Proceedings Archive, 34th International Conference on Biomechanics in Sport; 2016.

25. Jensen MM, Grønbæk K, Thomassen N, Andersen J, Nielsen J. Interactive footballtraining based on rebounders with hit position sensing and audio-visual feedback. Intern J Computer Science in Sport. 2014;13(1):57-68.

26. Antón Carranza M. Identificación del talento en la Organización: El Big Data aplicado al fútbol. 2017.

27. Holliday J. Effect of stroboscopic vision training on dynamic visual acuity scores: Nike Vapor Strobe® Eyewear. 2013.

28. Mitroff SR, Friesen P, Bennett D, Yoo H, Reichow AW. Enhancing ice hockey skills through stroboscopic visual training: a pilot study. Athletic Training and Sports Health Care. 2013;5(6):261-4.

29. Smith TQ, Mitroff SR. Stroboscopic training enhances anticipatory timing. International journal of exercise science. 2012;5(4):344. 
30. Wilkins L, Nelson C, Tweddle S. Stroboscopic Visual Training: a Pilot Study with Three Elite Youth Football Goalkeepers. Journal of Cognitive Enhancement. 2017:19.

31. Clark JF, Graman P, Ellis JK, Mangine RE, Rauch JT, Bixenmann B, et al. An exploratory study of the potential effects of vision training on concussion incidence in football. Optometry and Visual Performance. 2015;3(1).

32. Schwab S, Memmert D. The impact of a sports vision training program in youth field hockey players. Journal of sports science \& medicine. 2012;11(4):624.

33. Clark JF, Colosimo A, Ellis JK, Mangine R, Bixenmann B, Hasselfeld K, et al. Vision training methods for sports concussion mitigation and management. Journal of visualized experiments: JoVE. 2015(99).

34. Page JL. The BASES expert statement on the effectiveness of vision training programmes. The Sport and Exercise Scientist. 2013;38:12-3.

35. Wells AJ, Hoffman JR, Beyer KS, Jajtner AR, Gonzalez AM, Townsend JR, et al. Reliability of the dynavision ${ }^{\mathrm{TM}} \mathrm{d} 2$ for assessing reaction time performance. Journal of sports science \& medicine. 2014;13(1):145.

36. Peinado Palomin D, Torres Pareja M, García Coll MV, Mendoza Láiz N. Análisis del tiempo de reacción en personas con y sin discapacidad intelectual en función del deporte practicado. Cultura, Ciencia y Deporte. 2015;10(29).

37. Bigsby K, Mangine RE, Clark JF, Rauch JT, Bixenmann B, Susaret AW, et al. Effects of postural control manipulation on visuomotor training performance: comparative data in healthy athletes. International journal of sports physical therapy. 2014;9(4):436.

38. Ludvigsen M, Fogtmann MH, Grønbæk K, editors. TacTowers: an interactive training equipment for elite athletes. Proceedings of the 8th ACM Conference on Designing Interactive Systems; 2010: ACM.

39. Fogtmann $\mathrm{MH}$, Fritsch J, Kortbek KJ, editors. Kinesthetic interaction: revealing the bodily potential in interaction design. Proceedings of the 20th Australasian Conference on Computer-Human Interaction: Designing for Habitus and Habitat; 2008: ACM.

40. Jensen MM, Rasmussen MK, Grønbæk K, editors. Design sensitivities for interactive sport-training games. Proceedings of the 2014 conference on Designing interactive systems; 2014: ACM.

41. Politopoulos N, Tsiatsos T, Grouios G, Ziagkas E, editors. Implementation and evaluation of a game using natural user interfaces in order to improve response time. Interactive Mobile Communication Technologies and Learning (IMCL), 2015 International Conference on; 2015: IEEE.

42. Rozan MR, Sidik MKM, Sunar MS, Omar AH. KIHECTę: Reliability of Hand-Eye Coordination among Rugby Players Using Consumer Depth Camera. Computational Intelligence in Information Systems: Springer; 2015. p. 201-10.

43. Ellison PH, Sparks SA, Murphy PN, Carnegie E, Marchant DC. Determining EyeHand Coordination Using the Sport Vision Trainer: An Evaluation of Test-Retest Reliability. Research in Sports Medicine. 2014;22(1):36-48.

44. Ellison P, Jones C, Carnegie E. The Effect of Stroboscopic Vision Training on EyeHand Coordination. 2015. 
45. Vänttinen T, Blomqvist M, Luhtanen P, Häkkinen K. Effects of age and soccer expertise on general tests of perceptual and motor performance among adolescent soccer players. Perceptual and motor skills. 2010;110(3):675-92.

46. Vänttinen T, Blomqvist M, Häkkinen K. Development of body composition, hormone profile, physical fitness, general perceptual motor skills, soccer skills and on-theball performance in soccer-specific laboratory test among adolescent soccer players. Journal of sports science \& medicine. 2010;9(4):547.

47. Ward P, Williams AM. Perceptual and cognitive skill development in soccer: The multidimensional nature of expert performance. Journal of sport and exercise psychology. 2003;25(1):93-111.

48. Magner A, Chatham K, Spradley B, Wiriyapinit S, Price W, Akins T. Static stretching versus dynamic warm up: the effect on choice reaction time as measured by the Makoto Arena II. The Sport Journal. 2012;15(1).

49. Falcone PH, Tai C-Y, Carson LR, Joy JM, Mosman MM, Straight JL, et al., editors. Changes in cognitive function and sport-specific reaction time after active and passive dehydration. MEDICINE AND SCIENCE IN SPORTS AND EXERCISE; 2014: LIPPINCOTT WILLIAMS \& WILKINS 530 WALNUT ST, PHILADELPHIA, PA 191063621 USA.

50. Vacenovský P, Vencúrik T, editors. THE EFFECT OF SPECIFIC WARM-UP ON REACTIVE AGILITY IN TABLE TENNIS. 7TH INTERNATIONAL SCIENTIFIC CONFERENCE ON KINESIOLOGY.

51. Tai C-Y, Crowley KR, Spradley BD, Carson LR, Falcone PH, Esposito EN, et al. Reliability of an interactive sport-specific choice reaction time device. Journal of the International Society of Sports Nutrition. 2013;10(1):P18.

52. Junyent LQ, Fortó JS. Visió perifèrica: proposta d'entrenament. Apunts Educació física i esports. 2007;2(88):75-80.

53. Alves M, Spaniol F, Erichsen O. Visual skills of elite Brazilian soccer players. European Journal of Sports Science. 2014;4:1-9.

54. Baca A, Dabnichki P, Heller M, Kornfeind P. Ubiquitous computing in sports: A review and analysis. Journal of Sports Sciences. 2009;27(12):1335-46.

55. Wang JR, Parameswaran N, editors. Survey of sports video analysis: research issues and applications. Proceedings of the Pan-Sydney area workshop on Visual information processing; 2004: Australian Computer Society, Inc.

56. Bal B, Dureja G. Hawk eye: a logical innovative technology use in sports for effective decision making. Sport Science Review. 2012;21(1-2):107-19.

57. Burcham MA. Suppression of the Rotational Vestibulo-Ocular Reflex during a Baseball Pitch: The Ohio State University; 2010.

58. Harpham JA, Mihalik JP, Littleton AC, Frank BS, Guskiewicz KM. The effect of visual and sensory performance on head impact biomechanics in college football players. Annals of biomedical engineering. 2014;42(1):1-10.

59. Poltavski D, Biberdorf D. The role of visual perception measures used in sports vision programmes in predicting actual game performance in Division I collegiate hockey players. Journal of sports sciences. 2015;33(6):597-608.

60. Erickson GB. Give athletes a shot at better vision: whether they shoot foul shots or 
target rifles, your athletic patients require the best vision to stay at the top of their game. Review of Optometry. 2012;149(5):83-90.

61. De la Vega R, Almeida M, Ruiz R, Miranda M, Del Valle S. Entrenamiento atencional aplicado en condiciones de fatiga en fútbol. Revista Internacional de Medicina y Ciencias de la Actividad Física y del Deporte/International Journal of Medicine and Science of Physical Activity and Sport. 2011;11(42).

62. Maureira F, Bahamondes V, Jesam B, López R. Tiempo De Reacción Y Tiempo De Ejección En Arqueros De Fútbol Categoría Sub-15. El Físico de Chile. 2015:53-61.

63. Mora Mérida JA, Zarco Resa JA, Mena B, José M. Atención-concentración como entrenamiento para la mejora del rendimiento deportivo en jugadores profesionales de fútbol. Revista de psicología del deporte. 2001;10(1):0049-65.

64. de la Vega Marcos R. La importancia del entrenamiento de la concentración en el fútbol base: Una perspectiva aplicada. Cuadernos de Psicología del Deporte. 2003;3(2).

65. 65. De Rose L, editor Toma de Decisión en los Jugadores de Fútboli Víctimas o Victimarios? Una mirada crítica sobre las propuestas en el fútbol formativo. 11 Congreso Argentino de Educación Física y Ciencias 28 de septiembre al 2 octubre de 2015 Ensenada, Argentina; 2015: Universidad Nacional de La Plata. Facultad de Humanidades y Ciencias de la Educación. Departamento de Educación Física.

Referencias totales citadas: 65

Referencias citadas correspondientes a la Rev Ib CC Act Fis Dep: 0 